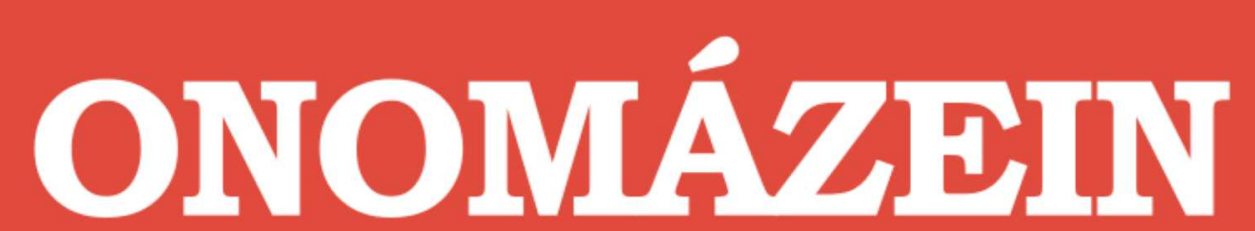

Revista semestral de lingüística, filología y traducción

\title{
Acerca de la transitividad de los procesos de comunicación en el discurso económico de divulgación argentino
}

On the transitivity analysis of communication processes

in Argentine economic media discourse

\author{
Juliana Montarcé \\ INCIHUSA - CONICET \\ Argentina
}

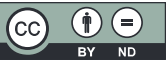

Juliana Montarcé: INCIHUSA - CONICET, Argentina. Correo electrónico: jmontarce@mendoza-conicet.gob.ar 


\section{Resumen}

En el marco de la Lingüística Sistémico-Funcional, siguiendo el modelo de Cardiff (Fawcett, 2000, 2008, 2011, en preparación a, en preparación b), abordamos la transitividad de los procesos de comunicación en el discurso económico de divulgación en lengua española. En la gramática de Cardiff, los procesos verbales son concebidos como subconjunto de procesos mentales de cognición y presentan una configuración de tres roles participantes: Agente + Fenómeno + Cognoscente-Afectado. El Agente da información o conocimientos a un Afectado, quien, a partir de ese momento, deviene en Cognoscente, y conoce así un Fenómeno. Se asume que esos tres roles son inherentes a este tipo de procesos, es decir, están exigidos por la semántica de los verbos aunque se encuentren encubiertos y no se realicen en el nivel de la forma (Fawcett, en preparación a, en preparación b).

En este trabajo, analizamos una submuestra (50 textos) de un corpus propio, denominado DIDIECO
(Discurso de Divulgación Económica), compuesto por textos de periódicos argentinos, conformado según postulados de la lingüística de corpus (Biber, 1993; McEnery y Wilson, 2001; Parodi, 2010). Realizamos el análisis sintáctico de todas las cláusulas y analizamos, mediante pruebas de verificación (Fawcett, 2011), los roles participantes requeridos por el proceso y su realización en el plano léxico-gramatical. Con el corpus anotado, nos proponemos así (i) establecer criterios de pertenencia a la clase "proceso de comunicación"; (ii) evaluar las probabilidades de instanciación de cada uno de los verbos en este tipo de discurso; (iii) pormenorizar la red sistémica propuesta por Fawcett, a partir de la concepción del "léxico como una gramática de mayor refinamiento" (Halliday, 1961), y (iv) considerar la diversidad de verbos que realizan procesos de comunicación a la luz del discurso en el que se insertan.

Palabras clave: Lingüística Sistémico-Funcional; gramática de Cardiff; procesos de comunicación verbal; discurso económico de divulgación.

\section{Abstract}

In the framework of Systemic Functional Linguistics, in the Cardiff model (Fawcett, 2000, 2008, 2011, forthcoming $a$, forthcoming $b$ ), we explore the transitivity system of communication processes in the economic media discourse in Spanish. In the Cardiff Grammar, verbal processes are conceived as a subset of mental processes of cognition and they have a configuration of three participant roles: Agent + AffectedCognizant + Phenomenon. The participant role Agent gives information or knowledge to an Affected who, thereafter, becomes Cognizant, and so comes to know of some Phenomenon. It is assumed that these three roles are inherent in this process type, i.e., they are required at the level of meaning, even if they are covert and sometimes unrealized at the level of form (Fawcett, in preparation $a$, in preparation b). In this paper, we analyze a subset (50 texts) of a corpus called DIDIECO (Economic Media Discourse), built according to the principles of Corpus Linguistics (Biber, 1993; McEnery \& Wilson, 2001; Parodi, 2010). In order to annotate the corpus, syntactic and semantic analysis were made, applying verification tests (Fawcett, 2011) for participants roles. Our main purposes are: (i) distinguish criteria to identify members of the class "communication process"; (ii) determine the frequency of instantiation of each verb in this kind of discourse; (iii) extend in delicacy the system network proposed by Fawcett, based on the concept of "lexis as most delicate grammar" (Halliday, 1961), and (iv) consider the diversity of verbs that realize communication processes in the light of the discourse in which they are inserted.

Keywords: Systemic Functional Linguistics; Cardiff Grammar; communication processes; economic media discourse. 


\section{Introducción}

El discurso periodístico constituye un espacio en el que diferentes voces confluyen, de manera armónica o bien con tensiones, para comentar, describir, analizar o evaluar algún fenómeno de la actualidad. Una parte importante del quehacer cotidiano de la noticia consiste en dar cuenta de lo que otros dicen. Numerosos son los autores que desde diferentes perspectivas han abordado la complejidad enunciativa de los textos periodísticos en lengua española (Reyes, 1982; Méndez García de Paredes, 1999; López Pan, 2002; Brunetti, 2009; Casado Velarde y De Lucas, 2013). Se ha señalado ya que en el discurso periodístico conviven códigos heterogéneos (lingüístico, tipográfico, icónico) y que el periodismo es en sí mismo una obliqua allocutio, esto es, un decir indirecto, puesto que se trata de un género textual integrado por diferentes formas de reproducir o referir el discurso ajeno. Brunetti (2009) explica claramente que el acto de referir el discurso ajeno consiste en una operación textual que se realiza poniendo en contacto, en el interior de un texto, dos acontecimientos lingüísticos: un contexto de enunciación 1 (en el que efectivamente se dijeron la palabras o enunciados que luego se citan) y un contexto de enunciación 2 (en el que el periodista refiere lo dicho en el contexto 1). Es importante destacar que las formas de reproducción del discurso son múltiples y variadas, con marcas explícitas o bien con indicios implícitos de polifonía textual, pero, en esta investigación, solo consideramos las instancias en las cuales existe un acto comunicativo en la situación de enunciación 1 que se reproduce en la situación de enunciación 2 mediante un proceso de decir.

Desde la Lingüística Sistémico-Funcional (en adelante, LSF), se ha observado la relevancia de los procesos verbales en los textos de la prensa (White, 1998; Halliday, 2004, 2014), en tanto le permiten al periodista atribuirle información a las fuentes. En el caso de las noticias económicas, observamos frecuentemente la atribución de discurso a especialistas en economía y a funcio- narios relacionados con el sector. Hemos constatado, en un proyecto de mayor alcance y desde una perspectiva de género, la fuerte presencia de reportes de discusión (issue report) (White, 1998) en la sección económica de los periódicos de nuestro corpus. Al tratarse de noticias que tienen como evento disparador un acto de habla, no es de extrañar que en el plano semántico observemos una fuerte presencia de procesos de comunicación y que dichos procesos se realicen, en el plano léxico-gramatical, a través de una gran cantidad y diversidad de verbos.

Sin dejar de considerar los aportes de la corriente predominante de la LSF, esta investigación aborda el análisis de la transitividad desde el modelo sistémico-funcional en su variante de Cardiff. Estimamos que provee no solo un marco descriptivo exhaustivo para el estudio de procesos y de roles participantes de la cláusula, sino también lineamientos para el análisis y un conjunto de pruebas que permiten verificar los resultados. Al trabajar con pruebas explícitas y criterios de significado, aspiramos a la posibilidad de replicar los resultados obtenidos.

Para este trabajo, nos hemos propuesto los siguientes objetivos: (i) establecer criterios de pertenencia a la clase "proceso de comunicación”; (ii) evaluar la frecuencia de instanciación de cada uno de los verbos en nuestro corpus; (iii) pormenorizar la red sistémica propuesta por Fawcett, a partir de la concepción del "léxico como una gramática de mayor refinamiento" (Halliday, 1961), y (iv) considerar la diversidad de verbos que realizan procesos de comunicación a la luz del discurso en el que se insertan.

\section{Marco teórico}

\subsection{La transitividad en la gramática de Cardiff}

La LSF aboga por una teoría que dé cuenta de cómo construimos nuestra experiencia del mundo que está dentro y alrededor nuestro (Halliday y Matthiessen, 1999: 11). Este modelo entiende la transitividad como la gramática de 
los procesos, es decir, de acciones y eventos, de procesos mentales y de relaciones, que pone en evidencia la forma en la que construimos un dominio particular de la experiencia (Halliday, 2004: 170).

El análisis de la transitividad consiste entonces en la identificación de procesos y de roles semánticos asociados. Dichos roles pueden ser de dos tipos: (i) roles participantes, requeridos por el proceso, que se realizan típicamente en la léxico-gramática como sujeto y complemento; y (ii) roles circunstanciales, que no están explícitamente exigidos por el proceso y que se realizan como adjuntos en la cláusula.

Desde la variante de Cardiff, Robin Fawcett (2008: 47) define la TRANSITIVIDAD como la columna vertebral del significado experiencial, pero su análisis se centra en los diferentes tipos de procesos y de roles participantes inherentes, mientras que los roles circunstanciales integran el significado experiencial de la cláusula, esto es, no los ubica dentro del sistema de TRANSITIVIDAD. Distingue, dentro de la LSF, dos enfoques diferentes para abordar su estudio: 1) distinguir tipos de situación (60 tipos de cláusulas) que las cláusulas realizan y 2) distinguir tipos de roles participantes (17 pruebas). Fawcett (en preparación a) arguye que, a los fines prácticos del análisis, es más conveniente comenzar por la identificación de roles participantes para determinar los diferentes tipos de situaciones ya que el número de pruebas de verificación que debemos efectuar es más acotado. En el plano teórico, son las diferentes configuraciones de roles participantes las que determinan los diferentes sentidos de una misma forma verbal. En la misma dirección, Neale (2002) sostiene que debemos proveer un análisis de la transitividad para cada una de las acepciones de un verbo.

Ahora bien, Fawcett (en preparación a: 6) aclara que para el reconocimiento de roles participantes no debemos atender a la forma del verbo, sino a su significado, es decir, a lo que la RAE (2009: 64) denomina su esqueleto de significación. Es la semántica la que indica cuáles son los roles participantes esperados para un determinado proceso, aunque no estén realizados en el nivel de la forma. Esta aseveración reviste particular importancia a la hora de diferenciar entre procesos mentales de cognición de dos roles, equivalentes a los procesos mentales de Halliday, de procesos mentales de cognición de tres roles, esto es, procesos de comunicación. No podemos dejar de mencionar que, al operar en el nivel del significado, es factible encontrar zonas grises o bien discrepancias en torno a la determinación de los participantes requeridos por el proceso. A pesar de todos los recaudos que toman las pruebas de verificación de Fawcett (2011, en preparación a, en preparación b) para evitar análisis de roles participantes ad hoc, nosotros disentimos con algunos análisis propuestos en la base de datos de tipos de procesos del inglés de Neale (2002), realizada desde la perspectiva de Cardiff. Por ejemplo, Neale analiza pray como un proceso mental de cognición de dos roles (Agent-Cognizant + Phenomenon) en "The men prayed for forgiveness", y lo define así: "speak to in order to give thanks or ask for help”. En cambio, postula que ask for en "I asked her for a glass of water" es mental de cognición de tres roles. Para nosotros, ambos procesos implican un tercer rol participante (el destinatario), a veces explícito y otras encubierto, es decir, no realizado en el nivel de la forma, por lo cual establecemos para los dos procesos la misma configuración de roles: Agent +Phenomenon + Affected-Cognizant. Este es tan solo uno de los casos de ambigüedad que se ponen de manifiesto al trabajar con un concepto problemático como el de "rol participante requerido por el proceso" ${ }^{\prime}$.

1 Nos cuestionamos acerca de lo que significa "estar requerido". En este sentido, existe una abundante bibliografía dedicada al estudio de argumentos y adjuntos. Si tan solo atendemos a la LSF, observamos que no existe consenso ni límites claros para distinguir roles participantes de roles circunstanciales. De hecho, Halliday (2014: 243) reconoce la existencia de un continuum en la relación participantes y circunstancias. 
Otro aspecto fundamental del análisis de la transitividad radica en el reconocimiento de los tipos de procesos como categorías no discretas (Matthiessen, 1995; Halliday, 2014: 216-218). Entendemos con Halliday (1995) que sistematizar no implica tomar decisiones categóricas, sino establecer proporcionalidades que reflejen el significado potencial en cuestión. El hecho de concebir los límites del sistema como indeterminados genera que, en algunos casos, veamos el mismo fenómeno dentro de una categoría o fuera de ella. Esto nos lleva a considerar la noción de grados de pertenencia a una clase, con miembros prototípicos y miembros periféricos; a cuestionarnos acerca de las consecuencias de clasificar de una manera o de otra, y a evaluar por qué un determinado proceso se encuentra en los límites de otro tipo de proceso.

\subsection{Los procesos de comunicación en la gramática de Cardiff}

Como adelantamos en el apartado anterior, los Ilamados procesos verbales se analizan, en la gramática de Cardiff, como una subcategoría de procesos mentales de cognición (Fawcett, 2008, 2011, en preparación a). Fawcett (en preparación b) aclara que su categoría de procesos de comunicación es similar² a la de Halliday pero un poco más amplia, ya que cubre otros códigos comunicativos y no solo el lenguaje. Añade que la principal diferencia reside en el hecho de que en Cardiff los procesos de comunicación se conciben como un subtipo de proceso mental mientras que, para la corriente predominante de la LSF, los procesos verbales constituyen un tipo de proceso independiente de los procesos de cognición.
El cuadro de la ilustración 1 muestra la red sistémica de TRANSITIVIDAD propuesta por Fawcett.

La configuración de roles participantes es la siguiente: Agente (Agent) + Fenómeno (Phenomenon) + Cognoscente-Afectado (Affected-Cognizant). La presencia del rol Cognoscente-Afectado es fundamental para distinguir los procesos de comunicación de los demás procesos mentales. Se asume que siempre hay un tercer rol participante, aun cuando está encubierto y no realizado en el nivel de la forma (Neale, 2002: 123).

Los procesos mentales de comunicación deben pasar una de las siguientes pruebas semánticas: (1) alguien le dice algo a alguien (tres roles participantes) o (2) el Agente ${ }_{1}$ hace que el Cognoscente-Afectado ${ }_{2}$ conozca algún Fenóme$\mathrm{no}_{3}$ (tres roles participantes). Al mismo tiempo, Fawcett (2011, en preparación a) propone pruebas específicas para el análisis de cada uno de esos tres roles:

(1) Si X es el Agente, la cláusula puede reformularse así: "Lo que hizo X fue...".

(2) Si X es el Afectado, la cláusula se reformula de la siguiente manera: "Lo que le sucedió a $X$ fue..."; si Y es el Cognoscente y X el Fenómeno, la cláusula se expresa así: "Y supo X por el Agente".

(3) Si Y es el Fenómeno y X el Cognoscente, podemos decir: "X supo Y por el Agente".

La categoría procesos de comunicación posee miembros prototípicos como decir o relatar, tal como se observa en [1] y en [2] de nuestro corpus $^{3}$.

2 Fawcett (en preparación b) sostiene: “If, despite these drawbacks, you still wished to follow Halliday's approach to Processes of this type while at the same time using the Cardiff Grammar approach to the other Process types, this would be possible".

3 Para ser más claros, hemos eliminado en los ejemplos del corpus las anotaciones correspondientes a las cláusulas que no son de comunicación. Entre corchetes, anotamos la función sintáctica combinada (con $\square$ ated) con el rol semántico. [S/Ag] corresponde a la función sintáctica de sujeto que desempeña el Agente; [V/Pro] significa ‘verbo de la cláusula que realiza el proceso' y, a su vez, este se va especificando con el uso de dos puntos; [C/F] representa el complemento que realiza el rol semántico Fenómeno; [C/Cog-Af] alude al complemento que se combina con el rol Cognoscente-Afectado. Cuando alguno de estos roles no está presente en el nivel de la forma pero sí se considera en el nivel semántico, se indica entre paréntesis: ([C/Cog-Af]). Los adjuntos se distinguen con Ilaves: \{\}. En los ejemplos que siguen aparecen el adjunto de tiempo y el adjunto de lugar, que se marcan así: \{A/T\} y $\{A / L u g\}$. 


\section{ILUSTRACIÓN 1}

Red sistémica de procesos de comunicación de Fawcett (en preparación a)

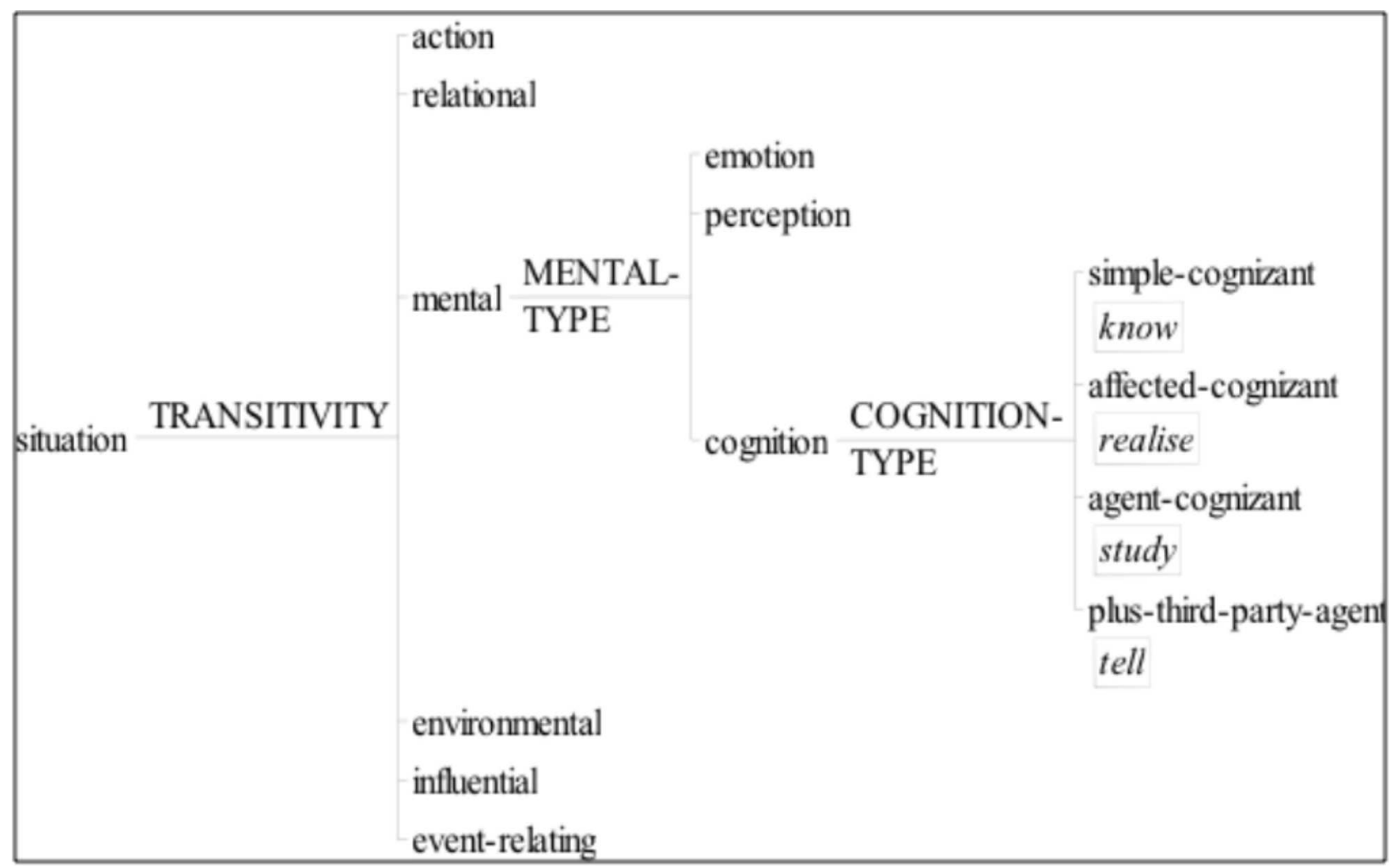

[1] el gerente de Asset Management y Research de Puente, Marcelo Olguín [S/Ag], dijo [V/Pro: mental: cognición: comunicación] a este diario [C/ Cog-Af] |que "cualquier turbulencia que reduzca el precio de los bonos complica el panorama"| [C/F]. (Página 12, 05/05/2010)

[2] Funcionarios que estuvieron presentes en la reunión [S/Ag] relataron [V/Pro: mental: cognición: comunicación] a PERFIL [C/Cog-Af] |que las expectativas de los empresarios estadounidenses se convertirán pronto en inversiones) [C/F]. (PerI, 10/04/2010),

y otros miembros periféricos, como se verá en 4.

Fawcett (en preparación b) juzga innecesario crear una categoría independiente por distintas razones. En primer lugar, es posible analizar los procesos verbales con los mismos roles participantes que intervienen en los procesos mentales; por lo tanto, no hay necesidad de complejizar el análisis agregando roles parti- cipantes específicos. Se agrega únicamente un Agente, con lo cual se evidencia mejor que comunicar es una forma de actuar. En segundo lugar, se pierde la valiosa generalización de que este tipo de proceso de tres roles es subsidiario de un proceso de cognición (decirle algo a alguien da como resultado que alguien sepa algo). Por otra parte, en un intento de simplificar el modelo sistémico-funcional, Fawcett propone el rol participante Fenómeno como una categoría amplia que cubre cuatro fenómenos: Verbiage, Target, cláusulas proyectadas con hipotaxis y cláusulas proyectadas con parataxis. Esas diferencias las interpreta como propiedades internas de la categoría Fenómeno.

Si se revisa la bibliografía referida al análisis de la transitividad, se observa que no existe consenso absoluto respecto del estatus de los procesos verbales ni de los criterios para identificarlos. Si bien esa problemática excede el alcance 
de este trabajo, tomaremos algunos aspectos que nos permiten dilucidar mejor nuestros criterios. En primer lugar, nos interesa recordar que Halliday (1970: 153) ubicaba los procesos que denominaba de verbalización dentro de los procesos mentales, junto con los de percepción, de reacción y de cognición. Sin embargo, en Introduction to Functional Grammar (2004, 2014) reconoce como procesos primarios los procesos materiales, relacionales y mentales. Los demás procesos - entre los que se encuentran los verbales - son secundarios en el sentido de que comparten rasgos con otros procesos pero constituyen una categoría independiente.

Arús (2003: 111) considera los procesos verbales como un grupo independiente "a pesar de la evidente familiaridad que presentan con los mentales". Luego de enumerar las similitudes con los procesos mentales, da como razón fundamental para separarlos el hecho de que una locución en un proceso verbal experimenta un doble proceso, primero se representa semánticamente y luego se recodifica mediante palabras, mientras que una idea en un proceso mental solo se procesa una vez, como significado. Nosotros estimamos que esa es una propiedad interna del Fenómeno y no una razón suficiente para identificar distintos procesos.

Martin, Matthiessen y Painter (1997: 120) sostienen que las cláusulas mentales son similares a las verbales en la selección del presente como tiempo no marcado y en la capacidad de proyectar otra cláusula. Pero lo que permite diferenciarlas es el hecho de que la cláusula verbal admite normalmente un receptor. La gramática de Cardiff, en cambio, opera en el nivel del significado y de los participantes requeridos por el proceso. En este sentido, se asume que un proceso de comunicación tiene siempre un receptor aunque no esté presente en el nivel de la forma. Halliday, en 1970, sostiene que la distinción entre roles obligatorios y opcionales nos ayuda a relacionar funciones transitivas con el sistema de tipos de cláusulas, y agrega: "As, however, this involves recognizing that an 'obligatory' ele- ment may in fact be absent, we shall use the term 'inherent' rather than 'obligatory'. An inherent function is one that is always associated with a given clause type even if it is not necessarily expressed in the structure of all clauses of that type" (1970: 150). Sin embargo, en IFG de 2014, dice: "There is always one participant, representing the speaker; there may also be an additional one representing the addressee" (Halliday, 2014: 303). En consonancia con Fawcett, estimamos que en este tipo de procesos el receptor, esto es, el Cognoscente-Afectado, es un participante inherente y, por lo tanto, debemos considerarlo en el análisis semántico aunque esté encubierto en la sintaxis. Es más, Fawcett (en preparación a, en preparación b) y Neale (2002) distinguen algunos roles participantes que no se realizan en la léxico-gramática. Como intentaremos demostrar en 4, nosotros consideramos que, a veces, el Cognoscente-Afectado se presenta, en el nivel de la forma, de manera no prototípica y, en algunos casos, no se realiza pero se infiere a través de otros elementos de la cláusula.

La consideración del "esqueleto de significación" de los procesos (y no su realización en la forma) nos lleva a replantear ciertos análisis ambiguos. Por ejemplo, Martin, Matthiessen y Painter (1997: 126) muestran que algunos verbos como show, indicate y suggest pueden funcionar en cláusulas verbales y en cláusulas relacionales de identificación: "She showed us that the substance was potassium" (told, convinced, verbal) frente a "The result showed that the substance was potassium" (meant, was, relacional). Nosotros sostenemos que ambos casos son procesos de comunicación y adherimos a la explicación que proporcionan Delbecque y Lamiroy (1999: 2021): el sujeto de persona constituye el caso no marcado para esta clase de verbos, pero al sujeto de persona se le pueden asimilar todos aquellos casos en los que un sujeto de cosa tiene una relación metonímica con un agente humano.

Halliday (2014: 304) también defiende la idea de que las cláusulas verbales no requieren de un participante consciente en función de sujeto y 
da los siguientes ejemplos: "The study says that such a diversified village structure produces a dualistic pattern of migration" y luego: "The letter says: 'It is observed that...". Sin embargo, ejemplos similares están usados también para explicar cláusulas relacionales de identificación bajo la subcategoría de demostración: "The fluorocarbon theory suggests that there should be a change..." (Halliday, 2014: 285). Llama la atención que no se especifique cuál sería el análisis más apropiado. Si bien reconocemos que, en los casos en los que no se realiza el tercer rol participante, el énfasis está puesto en la relación entre entidades, consideramos que existe, en la semántica, un tercer rol que puede reponerse y que se identifica con el destinatario del mensaje. Es por ello que, en nuestro análisis, consideramos procesos de comunicación casos como [3]:

[3] Los resultados de los últimos relevamientos [S/ $\mathrm{Ag}$ ] sugieren [V/Pro: mental: cognición: comunicación] una desaceleración de la actividad económica en los próximos meses [C/F] ([C/Cog-Af]) (Buenos Aires Económico, 05/10/2011).

Finalmente, nos interesa señalar otra diferencia con el análisis propuesto por Martin, Matthiessen y Painter (1997). Sostienen que el criterio central para distinguir un proceso verbal frente a uno de comportamiento es su capacidad de proyectar. Dan como ejemplo outline, describe, portray, como verbos que no admiten proyección. Sin embargo, al menos en lengua española, sí constatamos el uso de estos verbos con cláusulas proyectadas, fundamentalmente con citas directas.

\subsection{El proceso comunicativo y el rol participante Fenómeno}

El rol participante Fenómeno, que cumple la función sintáctica de complemento, puede llenarse (be $\square$ led) con un grupo nominal, que es en general un evento, como en [4], o bien en la mayoría de los casos se llena con un reporte de un texto, que puede darse de manera directa [5] o indirecta $[6]^{4}$.

[4] \{Una semana después de asumir su cargo\} $[\mathrm{A} / \mathrm{T}]$, el primer ministro Pedro Passos Coelho [S/Ag] anunció [V/Pro: mental: cognición: comunicación] una venta generalizada de empresas públicas [C/F] ([C/Cog-Af]). (Tiempo Argentino, 29/06/2011)

[5] |Brasil estableció un impuesto sobre operaciones financieras (IOF) del 6\% ... [C/F], anunció [V/ Pro: mental: cognición: comunicación] el ministro de Hacienda, Guido Mantega [S/Ag] ([C/CogAf]). (Tiempo Argentino, 30/03/2011)

[6] El BCE [S/Ag] anunció [V/Pro: mental: cognición: comunicación] lque comprará bonos en el mercado para blindar a Italia y Españal [C/F] ([C/CogAf]). (El Economista, 15/08/2011)

Fawcett (en preparación a, en preparación b) sugiere los siguientes análisis en los que reportes Ilenan el Fenómeno, en función de complemento ${ }^{5}$. En el reporte directo, la cláusula llena una sentencia, que es un elemento del texto, $y$, junto con las comillas, Ilenan el Fenómeno. En cambio, en el reporte indirecto, el Fenómeno se Ilena con una o más cláusulas, tal como muestran los gráficos de la ilustración 2.

En el caso de los reportes indirectos, la condición semántica general para que el complemento adquiera la forma de una subordinada sustantiva es que el verbo exprese un proceso cognitivo (Delbecque y Lamiroy, 1999: 1996).

En ambos tipos de reportes, el verbo funciona como expresión introductora o marco y proporciona el contexto adecuado para interpretar las palabras reproducidas (Méndez García de Paredes, 1999). El verbo introductor de la cita directa o indirecta enriquece el acto de habla que se cita al agregarle diferentes matices al verbo,

4 En realidad, las formas de referir el discurso ajeno son integrantes de un continuum, cuyos polos son directo e indirecto, pero en el medio encontramos muchas formas híbridas. No nos detendremos aquí a analizar estas diferencias, solo destacaremos algunas características generales.

5 Por razones de espacio, el análisis de nuestro corpus muestra las categorías sintácticas y semánticas entre corchetes 


\section{ILUSTRACIÓN 2}

Reporte directo y reporte indirecto

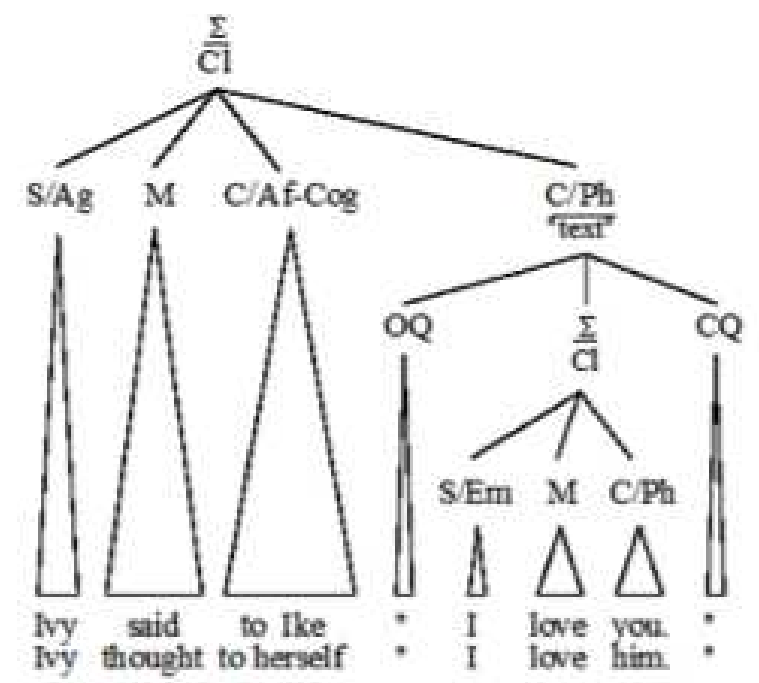

tal como demuestran Casado Velarde y De Lucas (2013). La gran variedad de ítems léxicos capaces de desempeñar esta función da cuenta del gran potencial de la lengua para proyectar.

\section{Metodología}

El estudio se llevó a cabo con un corpus textual propio, denominado DIDIECO (Discurso de Divulgación Económica), constituido según postulados de la lingüística de corpus (Biber, 1993; McEnery y Wilson, 2001; Parodi, 2010). Está compuesto de 300 textos publicados entre 2010 y 2011 en la sección económica de los periódicos argentinos La Nación, Clarín, Página 12, Per $\square$ y Tiempo Argentino, y en diarios especializados de economía como Buenos Aires Económico, El Cronista Comercial y Ámbito Financiero. Para este trabajo, seleccionamos al azar una muestra de 50 textos, con un promedio de 800 palabras por texto, y realizamos el análisis sintáctico y semántico de cada una de sus cláusulas, aplicando las pruebas propuestas por Fawcett (2011, en preparación a, en preparación b). Con el resultado del análisis procedimos a la anotación semántica y sintáctica del corpus y conformamos una base de datos con las diferentes instancias de procesos, entre ellos los de comunicación. Distin-

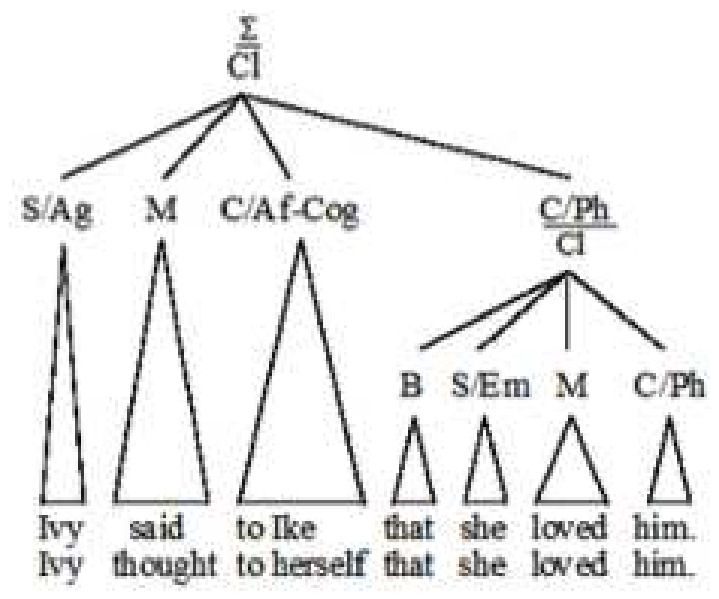

guimos instancias que contienen una cláusula proyectada (discurso directo, indirecto y sus variantes) de aquellas que llenan el Fenómeno con un grupo nominal. Al mismo tiempo, señalamos el orden en que los roles semánticos se realizan en la sintaxis.

De acuerdo con lo expuesto en 2, adoptamos como criterio de identificación de procesos de comunicación el hecho de que superaran exitosamente las pruebas generales del proceso así como la de cada uno de los roles participantes.

El hecho de trabajar con un corpus textual y no con ejemplos creados aumenta la complejidad del análisis ya que encontramos usos no convencionales, una gran diversidad de ítems léxicos, cláusulas extensas, un gran número de cláusulas proyectadas y muchos niveles de subordinación. Pero nos aporta la gran ventaja de analizar instancias auténticas de uso de la lengua y de recurrir a la inferencia textual y contextual. Nos interesa destacar que no hemos trabajado con un procedimiento habitual como es el de operar con una lista preestablecida de verbos y realizar las búsquedas correspondientes en el corpus; por el contrario, hemos analizado y anotado todas las cláusulas del corpus. 


\section{Análisis y discusión}

En los 50 textos analizados, detectamos 425 instancias de procesos de comunicación, realizados en el plano léxico-gramatical a través de 97 verbos diferentes. La ilustración 3 muestra la gran cantidad y diversidad de verbos empleados para este tipo de proceso en el discurso de divulgación económica. Tal como se observa en el cuadro, el verbo decir es el más empleado, pero solo representa el $11 \%$ del total, seguido por señalar, con el $5 \%$; agregar, advertir constituyen el $4 \%$; $a$ rmar, asegurar, conside- rar, informar, el $3 \%$, y el resto de las instancias representan un porcentaje aún menor. De las 425 cláusulas de comunicación, encontramos 342 que proyectan y 83 que se instancian sin proyección. De las cláusulas proyectadas, 143 introducen discurso directo; 122, discurso indirecto, y 68, un discurso mixto o pseudodirecto (Maldonado González, 1999; Brunetti, 2009).

En el cuadro de la ilustración 4, mostramos la totalidad de verbos relevados en el corpus con la cantidad de instancias de cada uno de ellos.

\section{ILUSTRACIÓN 3}

Frecuencia de instancias de verbos de comunicación en el corpus

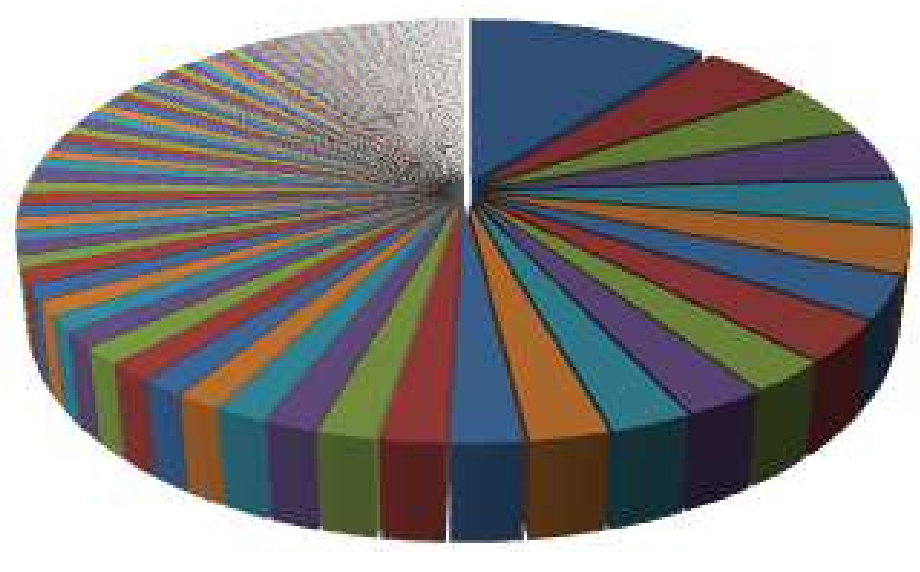

\begin{tabular}{l|l} 
decir & $11 \%$ \\
\hline señalar & $5 \%$ \\
\hline sostener & $4 \%$ \\
\hline agregar & $4 \%$ \\
\hline advertir & $4 \%$ \\
\hline afirmar & $3 \%$ \\
\hline asegurar & $3 \%$ \\
\hline considerar & $3 \%$ \\
\hline informar & $3 \%$
\end{tabular}

\section{ILUSTRACIÓN 4}

Instancias del corpus

\begin{tabular}{|c|c|}
\hline $\begin{array}{l}\text { Cantidad de } \\
\text { instancias }\end{array}$ & Verbos \\
\hline $\begin{array}{l}\text { Verbo más } \\
\text { empleado }\end{array}$ & decir (47) \\
\hline $\begin{array}{l}\text { Entre } 10 \text { y } 20 \\
\text { instancias }\end{array}$ & $\begin{array}{l}\text { señalar (20), sostener (18), agregar (17), advertir (16), indicar (15), afirmar (14), asegurar (14), anunciar (11), } \\
\text { considerar (11), explicar (11), informar (11), }\end{array}$ \\
\hline $\begin{array}{l}\text { Entre } 1 \text { y } 10 \\
\text { instancias }\end{array}$ & 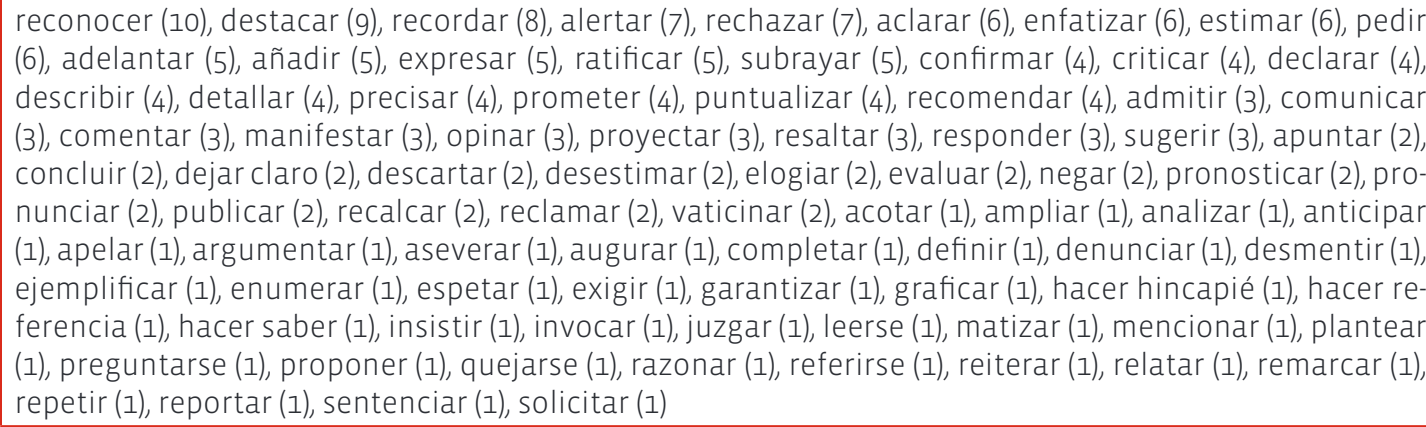 \\
\hline
\end{tabular}


Si observamos el ejemplo [7], vemos que aparece el verbo prototípico de la clase comunicación con sus roles participantes inherentes (Agente + Cognoscente-Afectado + Fenómeno):

[7] \{La semana pasada\} [A/T], el viceministro de Economía, Roberto Feletti [S/Ag], dijo [V/Pro: mental: cognición: comunicación] a Tiempo Argentino [C/Cog-Af] \{en una entrevista\} [A/Lug] |que "no nos parece que a un país tan solvente, y hoy la Argentina lo es, se le imponga como condición la revisión del FMI, como si fuéramos un país deudorl [C/F]. (Página 12, 05/05/2010)

Al aplicar las pruebas de verificación, vemos que las supera sin dificultad:

[7a] Feletti ${ }_{1}$ le dice "no nos parece que a un país..." al periódico Tiempo Argentino (tres roles participantes).

[7b] Si Feletti es el Agente: "Lo que hizo Feletti fue decir que no les parece que un país...". Si Tiempo Argentino es el Afectado: "Lo que le sucedió a Tiempo Argentino fue saber que a Feletti no le parece...";

si $Y$ es el Cognoscente y $X$ el Fenómeno, la cláusula se expresa así: "Tiempo Argentino supo por Feletti que no le parece que un país...".

Si Y es el Fenómeno y X el Cognoscente: "Tiempo Argentino supo por Feletti que no le parece que un país....".

Ahora bien, se observa que en la mayoría de las instancias el tercer rol participante, esto es, el Cognoscente-Afectado, se encuentra encubierto, tal como refleja [8] con el uso de paréntesis en la anotación:

[8] |"Las nuevas quiebras son parte de la crisis de 2008"| [C/F], dijo [V/Pro: mental: cognición: comunicación] Gray [S/Ag] ([C/Cog-Af]), \{al descartar nuevos motivos para preocuparse $[\mathrm{A} / \mathrm{T}]$. (La Nación, 18/08/2010)

En [9], identificamos una forma no prototípica de realización del Cognoscente-Afectado que presenta la estructura ante + grupo nominal:

[9] |“La Argentina cuida los puestos de trabajo y a las empresas que invierten en el país"| [C/F],
([S/Ag]) dijo [V/Pro: mental: cognición: comunicación] ante 200 ejecutivos de empresas de los EE.UU [C/Cog-Af]. (Perロl, 10/04/2012)

El texto nos brinda la información necesaria para el análisis de esta cláusula: la presidente argentina dialoga en Washington con ejecutivos de empresas. Al aplicar la prueba para el tercer rol, vemos que la supera sin dificultad:

[9a] Af: Lo que le sucedió a los 200 ejecutivos de empresas fue saber (enterarse de) que la Argentina cuida... / Cog: 200 ejecutivos de empresas supieron que la Argentina cuida...

Podríamos preguntarnos entonces acerca de la diferencia entre ante 200 ejecutivos y a 200 ejecutivos. Consideramos que la preposición ante aporta además una noción de lugar al proceso, pero ello no impide identificar a los ejecutivos como los destinatarios del mensaje de la presidente.

Encontramos en el corpus algunos procesos de comunicación tales como sostener, enfatizar, considerar, juzgar, acotar que no admiten con naturalidad la realización del Cognoscente-Afectado, tal como se observa en [10], [11], [12] y [13]:

[10] Ambrosetti [S/Ag] sostuvo [V/Pro: mental: cognición: comunicación] |que "contribuiría a equilibrar la rotación de los cultivos y eso favorecería al sustento del suelo", por el incentivo de no tener que afrontar las alícuotas| [C/F] ([C/Cog-Af]). (La Nación, 02/08/2010)

[11] |“Estructuralmente, Argentina por varios años tendrá que ir desactivando el retraso en las tarifas y las presiones ya construidas y habrá una inflación alta. Argentina va camino a ser un país caro"| [C/F], enfatizó [V/Pro: mental: cognición: comunicación] ([S/Ag]) ([C/Cog-Af]). (Per $\square$, 02/10/2010)

[12] |“El consumo se viene manteniendo relativamente fuerte, incluso durante la recesión del año pasado, a partir de pilares relativamente sólidos, como la recuperación del empleo, y otros no tan sólidos, como el estímulo dado por la política monetaria y fiscal, unido a algún refugio contra la inflación”| [C/F], consideró [V/Pro: mental: cog- 
nición: comunicación] Maximiliano Castillo CarriIlo, de ACM [S/Ag] ([C/Cog-Af]). (Per $\square$, 18/07/2010)

[13] |"Esperemos que todo mejore, aquí no tenemos nada más que hacer"| [C/F], juzgó [V/Pro: mental: cognición: comunicación] el economista Jorge Todesca, de la consultora Finsoport [S/Ag] ([C/ Cog-Af]). (La Nación, 11/08/2010)

Si bien no hemos detectado la estructura sostenerle algo a alguien (*Ambrosetti sostuvo a los periodistas que...) ni tampoco con los otros verbos mencionados, los ejemplos [10], [11], [12] y [13] admiten la construcción ante + grupo nominal (Ambrosetti sostuvo ante los periodistas que...), que Ilenaría el Cognoscente-Afectado. De todas maneras, al recurrir al texto completo descubrimos que el tercer rol se infiere por otros elementos; en [10] el acto de habla de Ambrosetti constituye una respuesta a "especialistas consultados por La Nación" y en [11] a "según los analistas consultados por PERFIL”, lo cual refleja que son fragmentos de discurso reproducido del diálogo entre el periodista y el especialista en economía. Además de que es factible recuperar el rol Cognoscente-Afectado por el texto, todos los verbos están empleados como equivalentes de decir y serían reemplazables por el verbo prototípico.

Los verbos como considerar y recordar poseen diferentes sentidos según el uso que se les dé en diferentes cláusulas'. Por ejemplo, tienen el significado de 'pensar, meditar, reflexionar algo con atención y cuidado' y ‘traer a la memoria' respectivamente en cláusulas mentales de cognición que presentan dos roles participantes: Cognoscente + Fenómeno. Ahora bien, en el contexto de un reporte periodístico que gira en torno a la discusión y análisis de un hecho o de una afirmación, se actualiza más bien la idea de 'transmitirle algo a alguien', por lo cual una interpretación más acertada requiere de un tercer rol participante (Agente + Fenómeno + Cognoscente-Afectado). Tal como observamos en [14], se lo utiliza como un equivalente de decir:
[14] |“El impacto está más relacionado con las expectativas sobre lo que suceda posteriormente al canje"| [C/F], consideró [V/Pro: mental: cognición: comunicación] ante Página/12 [C/Cog-Af] Sabrina Corujo, jefa de Research de la consultora Portfolio Personal [S/Ag]. (Página 12, 05/05/2010)

Otros casos en los que podemos cuestionarnos acerca de la presencia del rol CognoscenteAfectado en el "esqueleto de significación" aparece con los verbos agregar, añadir, puntualizar, completar y ampliar, tal como se muestra en [15], [16], [17], [18], [19]:

[15] ([S/Ag]) Agregó [V/Pro: mental: cognición: comunicación] lque con las medidas "no habrá duda" sobre el cumplimiento de la meta de déficit del 2011 del endeudado país) [C/F] ([C/CogAf]). (Ámbito Financiero, 12/03/2011)

[16] |“También hubo algunas compras por parte del Gobierno en el sector de los bancos como, por ejemplo, Hipotecario (+6,99\%)"| [C/F], añadió [V/ Pro: mental: cognición: comunicación] ([S/Ag]) ([C/Cog-Af]). (El Cronista Comercial, 02/07/2010)

[17] |“ Brasil, por ejemplo, también padecerá la salida de capitales que buscarán lugares más seguros como, por ejemplo, el oro"| [C/F], puntualizó [V/Pro: mental: cognición: comunicación] ([S/Ag]) ([C/Cog-Af]). (El Cronista Comercial, 02/07/2010)

[18] |“Pero esta vez es una burbuja de deuda soberana”| [C/F], completó [V/Pro: mental: cognición: comunicación] ([S/Ag]) ([C/Cog-Af]). (El Cronista Comercial, 02/07/2010)

[19] Y ([S/Ag]) amplió [V/Pro: mental: cognición: comunicación] ([C/Cog-Af]): |"Ambos grupos unieron esfuerzos para boicotear la reapertura del canje"I [C/F]. (La Nación, 20/01/2010)

La base de datos ADESSE clasifica agregar como material: espacio: unión. Está definido de la siguiente manera: una entidad I se suma a una entidad 2. Ahora bien, cuando las entidades en cuestión son fragmentos de discurso, nos parece más relevante considerar una acepción del verbo como miembro de la clase comunicación, ya que refleja mejor la semántica del proceso.

6 Halliday (2014: 257) explicita diferentes sentidos del verbo remind, según su aparición en una cláusula mental o verbal. 
De hecho, para que un emisor pueda enunciar el verbo agregar, es necesario que antes haya explicitado en su discurso al menos una vez un verbo como decir o a $\square$ rmar; por lo tanto, el tercer rol participante se recupera de ese contexto, tal como sucede en [15]:

[15] |"Como precaución adicional para el 2011, las medidas de consolidación se fortalecerán, permitiéndonos tener un efecto adicional del 0,8 por ciento (del PIB)" | [C/F], afirmó [V/Pro: mental: cognición: comunicación] ([S/Ag]) a la prensa [C/ Cog-Af] \{en Lisboa\} [A/Lug]. (Ámbito Financiero, 12/03/2011)

Sin embargo, cabe destacar que puntualizar, completar y ampliar no explotan todo el potencial de los verbos de comunicación porque no se emplean con frecuencia en discurso indirecto.

Numerosos verbos que no son prototípicamente de comunicación se utilizan en los periódicos para introducir discurso directo y serían perfectamente reemplazables por el verbo decir. Observamos que esta gran diversidad responde a la necesidad de evitar repeticiones. Méndez García de Paredes (1999: 109) analiza la expresión fue desgranando un durísimo rosario de imputaciones y afirma: "Este verbo no es en primera instancia un verbo de comunicación, pero por insertarse en un enunciado de DR [discurso referido] pasa a funcionar como tal". Sostiene que se comporta como un verbo elocutivo que indica la manera de enunciar del locutor origina- rio y "precisamente, el grupo de elocutivos es la vía abierta en el paradigma de los verbos de comunicación por donde penetran nuevos elementos empleados metafóricamente”. En nuestro corpus, hallamos, entre otros ejemplos, [20] y [21]:

[20] |El problema por los tipos cambiarios no es «un duelo en un western»| [C/F], matizó [V/Pro: mental: cognición: comunicación] el ministro surcoreano de Finanzas, Yoon Jeung-Hyun [S/Ag] ([C/ Cog-Af]). (Ámbito Financiero, 22/10/2010)

[21] |“Muchos (ejecutivos) estamos preocupados por la inflación y los altos costos internos: no sólo por la carne sino también por la mano de obra”| [C/F], espetó [V/Pro: mental: cognición: comunicación] Woods Staton, presidente de Arcos Dorados-McDonald's, [S/Ag] frente a unos 200 ejecutivos de firmas de primera línea [C/Cog-Af].

(Per $\square$, 02/10/2010)

Todos los verbos detallados en el cuadro superan las pruebas propuestas por Fawcett para adjudicarse un lugar en la categoría de verbos de comunicación, aunque no todos lo hacen de la misma manera: existen miembros prototípicos y miembros periféricos. En este sentido, nos parece sumamente relevante la propuesta de un abordaje topológico de los procesos (Martin, Matthiessen y Painter, 1997), para dar cuenta de los rasgos que comparten con otros tipos de procesos. En la ilustración 5, ejemplificamos el grado de pertenencia a la clase de algunos verbos?

\section{ILUSTRACIÓN 5}

Grados de pertenencia a la clase, con miembros prototípicos y periféricos

+ Pensamiento

augurar / vaticinar / opinar / analizar

+ Acción

exigir / pedir / reclamar / criticar alertar / advertir / denunciar
+ Relación

añadir / completar / resaltar adelantar / subrayar
COMUNICACIÓN

decir

informar / expresar / relatar

reportar / comentar / mencionar / sostener

7 Dejamos para futuras investigaciones el estudio detallado de los rasgos comunes de esas regiones limítrofes. 
La mayoría de los verbos comunicativos aporta información sobre el acto lingüístico que se lleva a cabo. La pormenorización de la red sis- témica que proponemos en la ilustración 6 constituye un intento de especificar el tipo de información.

\section{ILUSTRACIÓN 6}

Pormenorización de la red sistémica de procesos de comunicación de Fawcett

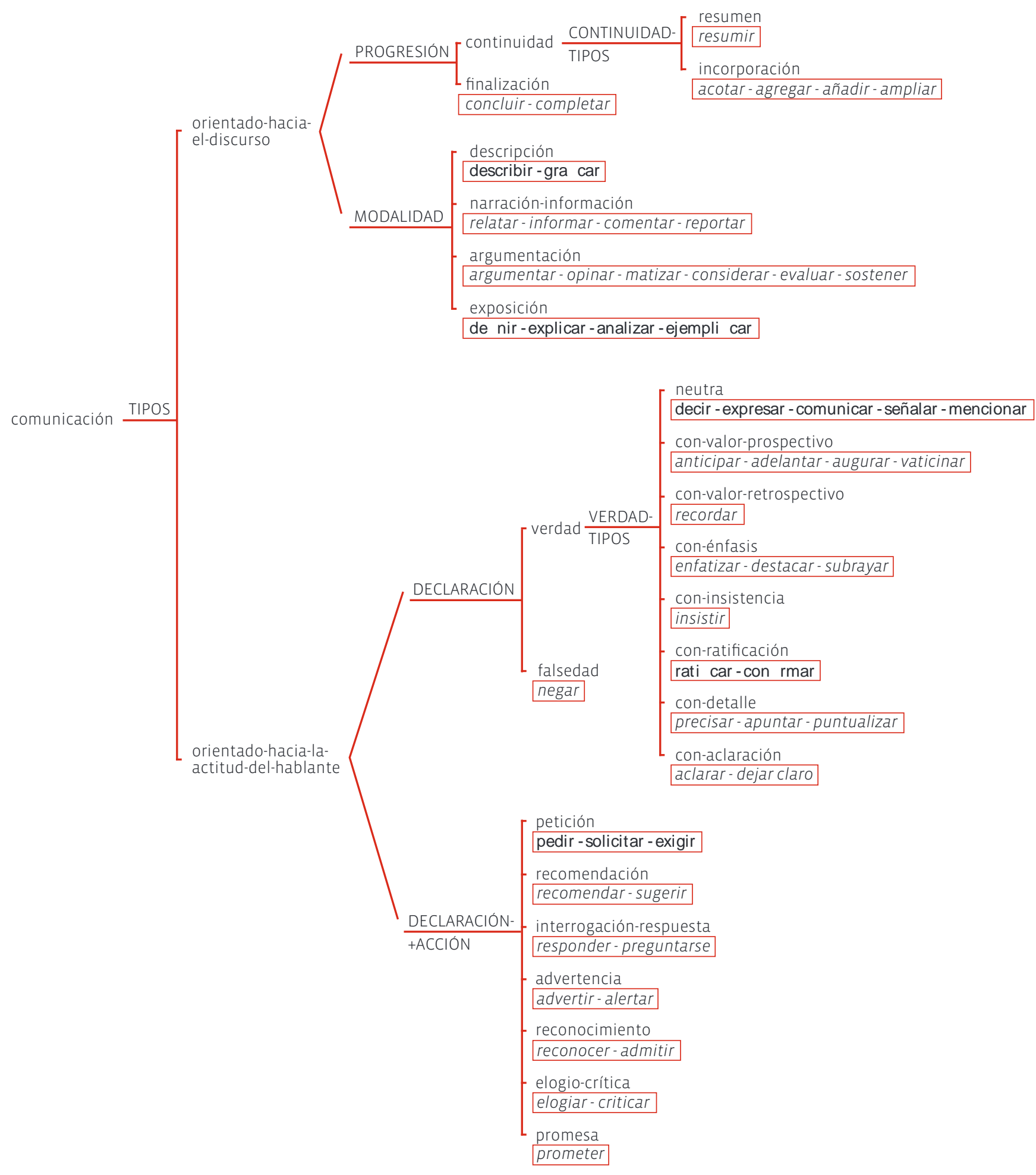




\section{Conclusiones}

En tanto complejos polifónicos, los textos periodísticos presentan numerosas instancias de reproducción del discurso ajeno. Los textos que componen nuestro corpus constituyen, en su gran mayoría, reportes de discusión en los que el evento disparador de la noticia es un acto de habla de un funcionario o de especialistas en materia económica. Ello explica no solo la cantidad de cláusulas comunicativas, sino también la gran diversidad de ítems léxicos que realizan los procesos de comunicación y que permiten evitar la repetición de verbos prototípicos como decir o a $\square$ rmar. Para realizar nuestro análisis, hemos justificado la conveniencia de operar con las categorías propuestas por la gramática de Cardiff y hemos aplicado las pruebas de verificación de roles participantes con el fin de determinar cuáles son los verbos que realizan ese tipo de procesos mentales de cognición. Hemos demostrado que los procesos de comunicación presentan siempre la siguiente configuración de roles participantes inherentes: Agente + Fenómeno + Cognoscente-Afectado, aun cuando alguno de sus participantes esté encubierto en el nivel de la forma. Hemos constatado también que la realización del tercer rol participante, es decir, del Cognoscente-Afectado, no es frecuente, pero que otros elementos de la cláusula y del texto permiten su inferencia. En cuanto al Fenómeno, observamos que puede llenarse con un grupo nominal, pero se llena más frecuentemente con sentencias y cláusulas en reportes directo e indirecto.

Propusimos también una red sistémica más detallada para los tipos de procesos de comunicación con la intención de reflejar que las distintas opciones del sistema no solo implican transferencia de información, sino también que les agregan matices y valoración a los procesos. Por otra parte, hemos apenas esbozado una organización topológica de este tipo de procesos para mostrar que los miembros periféricos se encuentran en los límites de otras categorías de procesos. Vemos así la posibilidad de explorar detalladamente, en posteriores investigaciones, cuáles son los rasgos semánticos que comparten los verbos que están en las zonas fronterizas.

Al trabajar con un corpus auténtico, el análisis se torna más complejo, pero la perspectiva es más enriquecedora, puesto que nos da la posibilidad de realizar inferencias textuales y del contexto de situación. Al respecto, hemos advertido que muchos verbos no integrarían el conjunto de verbos de decir si no fuera por el contexto en el que se insertan. Avizoramos, para trabajos futuros, la necesidad de profundizar la relación contexto - tipo de proceso.

\section{Bibliografía citada}

ADESSE: Base de datos de verbos, alternancias de diátesis y esquemas sintáctico-semánticos del español [http://adesse.uvigo.es/, fecha de consulta: marzo de 2014].

Arús, Jorge, 2003: Hacia una especi $\square$ cación computacional de la transitividad en español. Estudio contrastivo con el inglés. Tesis doctoral, Universidad Complutense de Madrid.

BiBer, Douglas, 1993: "Representativeness in Corpus Design", Literary and Linguistic Computing, vol. 8, no. 4, 243-257

Brunetti, Paulina, 2009: El discurso referido. Formas canónicas y no canónicas de citación en la prensa diaria, Córdoba: Comunicarte.

CAsAdo Vel Arde, Manuel y Alberto de I uCAs, 2013: "La evaluación del discurso referido en la prensa española a través de los verbos introductores", Revista Signos. Estudios de Lingüística.

d el BeCque, Nicole y Béatrice I Amir oy, 1999: "La subordinación sustantiva: las subordinadas enunciativas en los complementos verbales" en Ignacio Bosque y Violeta demonte (dirs.): Gramática descriptiva de la lengua española, vol. 2, Madrid: Espasa, 1965-2081. 
FAw Cett, Robin, 2000: A Theory of Syntax for Systemic Functional Linguistics, Amsterdam/Philadelphia: John Benjamins.

Fawcett, Robin, 2008: Invitation to Systemic Functional Linguistics through the Cardiff Grammar.An extension and simpli $\square$ cation of Halliday's Systemic Functional Grammar, London: Equinox. Faw Cett, Robin, 2011: "Problems and Solutions in Identifying Processes and Participant Roles in Discourse Analysis", Annual Review of Functional Linguistics, vol. 3, Higher Education Press, 34-87.

FAw Cett, Robin, en preparación a: "Analyzing experiential meaning" en The Functional Semantics Handbook: Analyzing English at the level of meaning, London: Equinox.

FAw Cett, Robin, en preparación b: "How to Analyze Participant Roles-and so Processes-in English", draft of chapter 2 (forthcoming 2015) in The Functional Semantics Handbook: Analyzing English at the level of meaning, London: Equinox.

HAlidAy, M. A. K., 1961: "Categories of the theory of grammar", Word 17(3), 241-292.

HAlidAy, M. A. K., 1970: "Language structure and Language function" en John I yons (ed.): New Horizons in Linguistics, Harmondsworth: Penguin, 140-165.

HAlidAy, M. A. K., 1995: "Fuzzy Grammatics: a systemic functional approach to fuzziness in natural language", Proceedings of FUZZ-IEEE/IFES '95 (The International Joint Conference of the Fourth IEEE International Conference on Fuzzy Systems and the Second International Fuzzy Engineering Symposium, March 20-24, 1995, Yokohama).

HA I idAy, M. A. K., 2004: An Introduction to Functional Grammar, Revised by C. Matthiessen, London: Arnold.

HA I id Ay, M. A. K., 2014: Halliday's Introduction to Functional Grammar, revised by C. Matthiessen, London: Routledge.

HA I idAy, M. A. K. y Christian mAt t Hiessen, 1999: Construing Experience through Meaning. A Language-based approach to Cognition, London: Continuum.
I ópez pAn, Fernando, 2002: "Las citas directas en el periodismo escrito. Literalidad y objetividad a la luz de los estudios lingüísticos", Comunicación y Sociedad, vol. XV, n. ${ }^{\circ}$ 2, 79-93.

mA donAdo Gonzál ez, Concepción, 1999: "Discurso directo y discurso indirecto" en Ignacio Bosque y Violeta demonte (dirs.): Gramática descriptiva de la lengua española, vol. 3, Madrid: Espasa-Calpe, 3549-3595.

mArtin, J. R., Christian mAttHiessen y Clare pAinter, 1997: Working with Functional Grammar, London: Arnold.

mAt thiessen, Christian, 1995: "Fuzziness construed in language: a linguistic perspective", Proceedings of FUZZ/IEEE, Yokohama, 1871-1878.

mœenery, Tony y Andrew wil son, 2001: Corpus Linguistics, Edinburgh: Edinburgh University Press.

méndez Gar Gía de paredes, Elena, 1999: "Análisis de la reproducción del discurso ajeno en los textos periodísticos”, Pragmalingüística 7, 99-128.

neAl e, Amy, 2002: More Delicate TRANSITIVITY: Extending the PROCESS TYPE system networks for English to include full semantic classi $\square$ cations. Tesis doctoral, Cardiff University.

pArodi, Giovanni, 2010: Lingüística de corpus: de la teoría a la empiria, Madrid: Iberoamericana vervuert.

r eA AcAdemia español A, 2009: Nueva gramática de la lengua española, Madrid: Espasa Calpe.

$r$ eyes, Graciela, 1982: "El estilo indirecto en el texto periodístico", LEA: Lingüística Española Actual, vol. 4, n. ${ }^{\circ}$ 1, 1-22.

W Hite, P. R. R, 1998: "Telling Media Tales: the News Story as Rhetoric", manuscrito no publicado, Sydney: University of Sydney. 